\title{
FE65 Constitutes the Functional Link between the Low-Density Lipoprotein Receptor-Related Protein and the Amyloid Precursor Protein
}

\author{
Claus U. Pietrzik, ${ }^{1,2}$ Il-Sang Yoon, ${ }^{1}$ Sebastian Jaeger, ${ }^{2}$ Tracy Busse, ${ }^{1}$ Sascha Weggen, ${ }^{1}$ and Edward H. Koo ${ }^{1}$ \\ ${ }^{1}$ Department of Neurosciences, University of California, San Diego, La Jolla, California 92093, and ${ }^{2}$ Institute for Pathobiochemistry, University of Mainz, \\ 55099 Mainz, Germany
}

Increasing evidence has implicated the low density lipoprotein receptor-related protein (LRP) and the adaptor protein FE65 in Alzheimer's disease pathogenesis. We have shown previously that LRP mediates $\beta$-amyloid precursor protein (APP) processing and affects amyloid $\beta$-protein and APP secretion and APP-c-terminal fragment generation. Furthermore, LRP mediates APP processing through its intracellular domain. Here, we set out to examine whether this interaction is of direct or indirect nature. Specifically, we asked whether adaptor proteins such as FE65 influence the LRP-mediated effect on APP processing by forming a protein complex. In coimmunoprecipitation experiments, we confirmed the postulated APP-FE65 and the LRP-FE65 interaction. However, we also showed an LRP-FE65APP trimeric complex using pull-down techniques. Because FE65 alters APP processing, we investigated whether this effect is LRP dependent. Indeed, FE65 was only able to increase APP secretion in the presence of LRP. In the absence of LRP, APP secretion was unchanged compared with the LRP knock-out phenotype. Using RNA short interference techniques against FE65, we demonstrated that a reduction in FE65 protein mimics the LRP knock-out phenotype on APP processing. These results clearly demonstrate that FE65 acts as a functional linker between APP and LRP.

Key words: alzheimer; amyloid precursor protein; APP; lipoprotein; low-density lipoprotein receptor-related protein; LRP; FE65; trafficking

\section{Introduction}

Alzheimer's disease $(\mathrm{AD})$ is a progressive neurodegenerative disorder characterized by senile plaques and neurofibrillary tangles. The major pathological component of senile plaque is amyloid $\beta$-protein $(\mathrm{A} \beta)$, which is usually a 40 - or 42 -amino acid (aa) peptide. $\mathrm{A} \beta$ is generated by proteolytic cleavage by two enzymes ( $\beta$ - and $\gamma$-secretase cleavage) from a larger type I integral transmembrane glycoprotein termed $\beta$-amyloid precursor protein (APP). In another pathway, APP is cleaved within the $\mathrm{A} \beta$ region by a proteinase activity known as $\alpha$-secretase, which has been identified as a member of the $\alpha$-disintegrin and metalloproteinase ADAM family (for review, see De Strooper and Annaert, 2000). This gives rise to a secreted form of APP (APPs $\alpha)$ and prevents generation of an intact $A \beta$ polypeptide.

Low-density lipoprotein receptor-related protein (LRP) is a $600 \mathrm{kDa}$ transmembrane glycoprotein with multifunctional receptor properties mediating the internalization and degradation of many extracellular ligands, including $\alpha 2$-macroglobulin, apolipoprotein E, and Kunitz protease inhibitor-containing forms of

Received Dec. 11, 2003; revised March 16, 2004; accepted March 16, 2004.

This work was supported in part by Deutsche Forschungsgemeinschaft Grant PI379 (C.U.P.) and National Institutes of Health Grant AG12376 (E.H.K.).

Correspondence should be addressed to Dr. Claus U. Pietrzik, Institute for Pathobiochemistry, Johannes Gutenberg University of Mainz, 55099 Mainz, Germany. E-mail: pietrzik@uni-mainz.de.

DOI:10.1523/JNEUROSCI.5451-03.2004

Copyright $\odot 2004$ Society for Neuroscience $\quad$ 0270-6474/04/244259-07\$15.00/0
APP (Herz and Strickland, 2001). LRP and the aforementioned ligands are also genetically associated with $\mathrm{AD}$ and are found in senile plaques in brains of AD patients (Kang et al., 2000). LRP is cleaved in the trans-Golgi network by furin to generate a $515 \mathrm{kDa}$ $\alpha$-subunit and $85 \mathrm{kDa} \beta$-subunit (Herz et al., 1990), which remain associated in a noncovalent manner as they are routed to the cell surface (Herz et al., 1990; Willnow et al., 1996). Interestingly, we and others have shown that LRP modulates multiple steps in APP processing and trafficking, including the turnover of both full-length and C-terminal fragments of APP, secretion of APPs, internalization of cell surface APP, and the generation and release of $A \beta$ peptide (Ulery et al., 2000; Pietrzik et al., 2002). The effect of LRP on APP processing and $\mathrm{A} \beta$ generation was mapped to a seven amino acid region (-NPTYATL-) within the intracellular domain of LRP, a finding consistent with colocalization of these two molecules by fluorescence resonance energy transfer (FRET) (Kinoshita et al., 2001; Pietrzik et al., 2002).

The mechanism by which LRP regulates APP processing through its cytoplasmic domain is unknown. Although LRPAPP complexes can be detected (Rebeck et al., 2001), APP is not known to directly bind to LRP. However, the adaptor protein FE65, a family of three related members including Fe65L1 and Fe65L2, has been shown to interact and colocalize with both LRP and APP. This has led to the proposal that FE65 is an adaptor molecule that links LRP and APP, although such a trimeric complex has not been demonstrated biochemically. Importantly, it is 
not known whether this complex, if present, is required for APP processing. In this study, we showed that APP and LRP are indeed linked through FE65 in a multiprotein complex as hypothesized. Furthermore, the three molecules are necessary and sufficient for the formation of this complex in vitro. Using LRP-deficient cells, our studies suggest that either LRP or FE65 can be the limiting factor in this protein complex. Thus, either the absence of LRP or FE65 would perturb APP processing, presumably by abrogating formation of this complex.

\section{Materials and Methods}

Cell lines and cDNA constructs. Mouse fibroblasts deficient in LRP [LRP-/-; pseudomonas exotoxin $\mathrm{A}$ (PEA)] and corresponding LRPexpressing control cells (LRP+/-; PEA 10) were obtained from American Type Culture Collection (Rockville, MD) and transfected with full-length human APP751 as described previously (Pietrzik et al., 2002). LRP-deficient Chinese hamster ovary $(\mathrm{CHO})$ cell line (135-1) and corresponding CHO K1 control cells (kind gift from Dr. S. Leppla, National Institutes of Health, Bethesda, MD) were grown in $\alpha$ DMEM supplemented with $10 \%$ fetal bovine serum (Fitzgerald et al., 1995). HS683 neuroglioma cells, which stably overexpress wild-type human APP695, have been described previously (Weggen et al., 2003). FE65-pcDNA3 (kind gift from Dr. J. Buxbaum, Mount Sinai School of Medicine, New York, NY) and deletion construct lacking the last 169 aa of the C terminus of FE65, FE65 $\Delta$ phosphotyrosine interaction domain (PID2), were subcloned into the retroviral expression vector pLHCX (Clontech, Palo Alto, CA) and transferred into $\mathrm{GP}+\mathrm{E} 86$ packaging cell line. After infection with recombinant viruses, LRP - / - and LRP + / - mouse fibroblasts and HS683 cells were selected with hygromycin, and stable cultures were used for analysis without additional clonal selection. APP-C50-myc was generated by PCR and transiently transfected into $\mathrm{CHO} 13-5-1$ and $\mathrm{CHO}$ K1 cells using Fugene6 (Roche, Indianapolis, IN) as described previously (Weggen et al., 2003).

Antibodies. The polyclonal LRP C-terminal antibody (Ab) (1704), the APP C-terminal polyclonal Ab (pAb) (CT15), the APP midregion pAb (863) (Sisodia et al., 1993; Marquez-Sterling et al., 1997; Pietrzik et al., 2002), and monoclonal antibodies $1 \mathrm{G} 7$ and $5 \mathrm{~A} 3$, which react with the ectodomain of APP and the monoclonal A $\beta$ antibody 26D6, have been described previously (Koo et al., 1996; Pietrzik et al., 2002). The polyclonal FE65 Ab (5916) was generated by immunizing rabbits with a synthetic peptide corresponding to the last 15 amino acids of the cytoplasmic domain of human FE65 coupled to keyhole limpet hemocyanin. The pAb to the WW binding domain of FE65 was a kind gift from Dr. J. Buxbaum.

Immunoprecipitation and immunoblotting. To detect APPs, media conditioned by the respective cell lines for $24 \mathrm{hr}$ were collected for analyses and immunoprecipitated using the monoclonal APP ectodomain antibodies 1G7 and 5A3. One percent NP-40 cell extracts and immunoprecipitates were fractionated by SDS-PAGE in $4-12 \%$ Tris-glycine gels. In all cases, gel loading was normalized to total protein content in the cell extract or the corresponding cell extracts when medium samples were used. Western blotting was performed with the indicated antibodies and detected by enhanced chemiluminescence (Pierce, Rockford, IL). Quantitation of the chemiluminescence signal was performed with a CCD camera imaging system (GeneGnome; Syngene, Frederick, MD).

26D6 APP Ab was used for coimmunoprecipitation experiments. Cells were lysed in 1\% 3-[(3-cholamidopropyl)dimethylammonio]-1-propanesulfonate buffer. Extracts and corresponding immunoprecipitates (1:10 for Hs683 cells) were fractionated by SDS-PAGE in $4-12 \%$ Tris- glycine gels. Western blotting was performed with the indicated antibodies and detected by enhanced chemiluminescence (Pierce).

Metabolic labeling. Confluent cultures of APP751-transfected LRP - / - fibroblasts overexpressing FE65 were incubated with phorbol 12-myristate 13-acetate (PMA) or DMSO vehicle in methionine-free DMEM supplemented with $150 \mu \mathrm{Ci} / \mathrm{ml}\left[{ }^{35} \mathrm{~S}\right]$ methionine/cysteine for 30 min. Cells were chased for $6 \mathrm{hr}$, and APPs was immunoprecipitated from conditioned media with monoclonal antibodies 1G7-5A3. The immunoprecipitates were fractionated by SDS-PAGE $(4-12 \%$ Tris-Tricine NuPAGE gels) and exposed to either film or phosphorimaging for quantitation.

In vitro translation and glutathione S-transferase pull down. Recombinant glutathione S-transferase (GST) fusion proteins were generated by subcloning the last $43 \mathrm{C}$-terminal amino acids of APP and the last 100 C-terminal amino acids of LRP into pGEX4T-2 plasmid (Amersham Biosciences, Arlington Heights, IL). Bacterial expression of fusion proteins was induced with isopropyl- $\beta$-thiogalactopyranoside. Induced proteins were purified using glutathione-agarose beads according to manufacturer instructions (Amersham Biosciences). Affinity precipitation was performed as follows. Chaps-lysates were incubated with GSTagarose beads for $1 \mathrm{hr}$ at $4^{\circ} \mathrm{C}$. Subsequently, fusion proteins were added to precleared lysate and incubated for $1 \mathrm{hr}$ at $4^{\circ} \mathrm{C}$. Beads were washed three times in Chaps buffer, and bound LRP was identified by SDS-PAGE followed by immunoblotting.

Stabilization of APP-C50 by FE65. The APP C50-myc construct was cotransfected into $\mathrm{CHO} \mathrm{K} 1$ and $\mathrm{CHO} 13-5-1$ cells with either control plasmid or pCDNA3-FE65. Forty-eight hours after transfection, cells were extracted with NP-40-lysis buffer for $30 \mathrm{~min}$ and centrifuged at $20,000 \times g$ for $10 \mathrm{~min}$ at $4^{\circ} \mathrm{C}$. Insoluble material was re-extracted in $10 \mathrm{ml}$ of $1 \%$ SDS (Kimberly et al., 2001), sonicated, and then diluted to $0.1 \%$ SDS and cleared by centrifugation at $20,000 \times g$ at $4^{\circ} \mathrm{C}$ for $20 \mathrm{~min}$. NP-40 and SDS extracts were immunoprecipitated with monoclonal anti-myc $\mathrm{Ab}$ 9E10. Immunoprecipitated material was separated on $4-12 \%$ BisTris gels, and the APP C50-myc fragment was detected by Western blotting with pAb CT15.

\section{Results}

\section{FE65 complexes with APP and LRP}

In this study, we hypothesized that complex formation between LRP and APP is required for normal APP processing. To test for the presence of APP-FE65-LRP complexes, we first assayed for APP-FE65 and LRP-FE65 complexes. As expected, on the basis of 


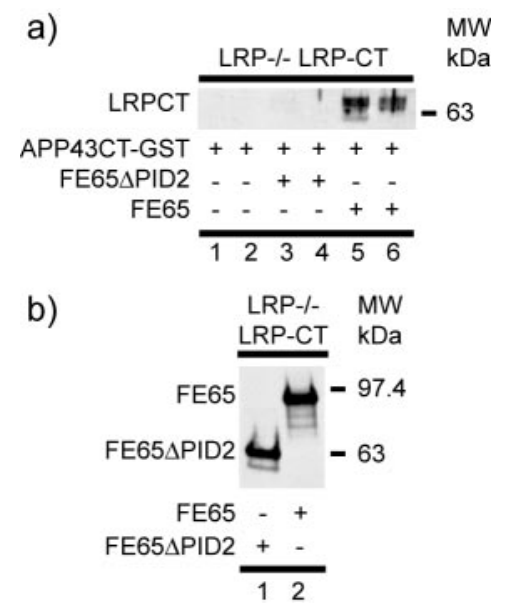

Figure 2. APP43CT-glutathione S-transferase pull-down of LRP-FE65 complex. $a$, Lysates from LRP-deficient mouse embryonic fibroblast (MEF) cells stably overexpressing LRP-CT were transfected with FE65 (lanes 5, 6) or FE65 $\Delta$ PID2 expression vector (lanes 3, 4) or untransfected controls (lanes 1, 2). Lysates were incubated with APP43CT-glutathione S-transferase. Bound proteins were coprecipitated by the addition of glutathione-agarose. Proteins were detected with polyclonal anti-LRP Ab 1704. Note that only in the presence of functional FE65 was the LRP-CT fragment pulled down by APP43CT-glutathioneS-transferase. The FE65 and FE65 $\Delta$ PID2 expression in LRP-deficient MEF cells stably overexpressing LRP-CT is shown in $b$ using an Ab against the WW domain in FE65. MW, Molecular weight.

published reports, APP-FE65 complexes can be immunoprecipitated from human embryonic kidney (HEK) 293 cells cotransfected with APP695 and FE65 (Fig. 1a). Similarly, FE65-LRP complexes can be detected in 293 cells expressing an $\mathrm{N}$-terminal truncated LRP construct, LRP-CT, and FE65 (Fig. 1b). The LRP cytoplasmic tail contains two Asm-Pro-X-Tyr NPXY motifs, and it has been suggested that the proximal site is the domain that interacts with FE65 (Trommsdorff et al., 1998). To test this hypothesis, deletions were generated in the $\mathrm{N}$-terminal truncated LRP-CT construct, deleting either NPXY motifs in the cytoplasmic domain of LRP, LRP-CT $\Delta 1$ and LRP-CT $\Delta 2$ (Pietrzik et al., 2002 ), or both (LRP-CT $\Delta 1 \Delta 2$ ). Surprisingly, after expression in 293 cells, FE65 can be coimmunoprecipitated with both LRPCT $\Delta 1$ and LRP-CT $\Delta 2$ but not LRP-CT $\Delta 1 \Delta 2$. This indicated that FE65 does not discriminate between these two NPXY motifs of LRP and can interact with both domains. It should be noted that in this experiment, we could detect interaction between endogenous LRP and the transfected FE65, because low levels of complexes can be immunoprecipitated in mock-transfected control and LRP-CT $\Delta 1 \Delta 2$-transfected cells (Fig. 1b). Comparable expression of the FE65 and the various LRP-CT constructs are shown in Figure $1 c$.

\section{Evidence for APP-FE65-LRP complex}

The above results are consistent with published reports suggesting that both LRP and APP bind to FE65. To test for the presence of trimeric complex consisting of APP, FE65, and LRP, GST-APP pull-down experiments were performed. For this analysis, LRP-/ - cells expressing LRP-CT were transfected with either FE65 or with a deletion mutant of FE65 lacking the second PID domain (FE65 $\Delta$ PID2). Deletion of the latter PID domain abrogates the interaction with APP (for review, see Russo et al., 1998). With this assay, the GST-APP construct was able to complex with LRP-CT only in the presence of FE65 (Fig. 2a), indicating that FE65 facilitated complex formation between APP and LRP. When FE65 was not transfected or when the mutant FE65 construct deleted the second PID domain expressed, there was no

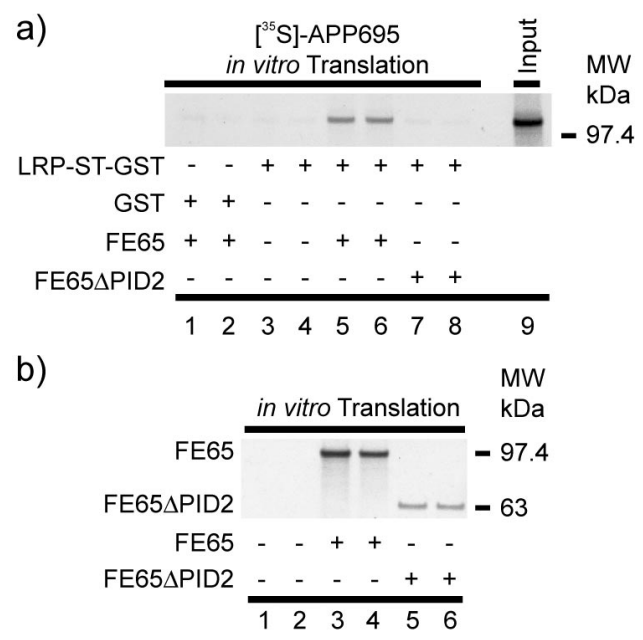

Figure 3. LRP-ST-glutathione S-transferase pull-down of in vitro translated FE65-APP695 complex. a, APP695 was in vitro translated in the presence of [ $\left.{ }^{35} \mathrm{~S}\right]$-Methionine and incubated with in vitro translated FE65 (lanes 5, 6) or FE65 PID2 (lanes 7, 8). As control, [ $\left.{ }^{35} \mathrm{~S}\right]$-APP695 was incubated without FE65 or FE65 $\triangle$ PID2 (lanes 3, 4). After incubation with LRP-STglutathione S-transferase fusion protein or GST fusion protein alone (lanes 1 and 2), bound proteins were coprecipitated by the addition of glutathione-agarose. Radiolabeled APP695 was detected by exposure to $x$-ray film, and the radioactive decays were quantified using phosphorimaging techniques. Note that only in the presence of functional FE $65, \sim 30 \%$ of the radiolabeled APP695 was pulled down by LRP-ST-glutathione S-transferase. The in vitro translated FE65 and FE65 $\Delta$ PID2 proteins used in these experiments are shown in $b$. MW, Molecular weight.

interaction between APP C-terminal fusion protein and LRP-CT (Fig. 2a). The expression levels of transfected FE65 and FE65 $\Delta$ PID2 are shown in Figure $2 b$.

The above results suggested that FE65 does indeed enhance APP-LRP interaction. Therefore, we next determined whether these three proteins are sufficient for the formation of a tripartite complex. In this experiment, a soluble LRP C-terminal fusion protein (LRP-ST-glutathione S-transferase) was used to pull down in vitro translated full-length APP695 labeled with [ $\left.{ }^{35} \mathrm{~S}\right]$-methionine in the presence or absence of in vitro translated FE65. Consistent with the notion that functional FE65 is necessary and sufficient for APP to complex with LRP, LRP-ST-glutathione $S$-transferase was able to precipitate radiolabeled APP695 only when full-length FE65 was present (Fig. 3). In the absence of FE65 or in the presence of FE65 $\Delta$ PID2, there was no interaction between LRP-ST-glutathione $S$-transferase and radiolabeled APP695. We estimated that $30 \%$ of the input APP695 can be precipitated by LRP-ST-glutathione $S$ transferase when FE65 is present. The expression levels of in vitro translated FE65 and FE65 $\Delta$ PID2 are shown in Figure $3 b$. Therefore, APP, FE65, and LRP together are necessary and sufficient to form a trimeric complex in vitro.

\section{LRP expression mediates FE65-induced APPs secretion}

Having demonstrated biochemically the interaction between APP, FE65, and LRP, we next asked whether this tripartite complex has any functional role in APP processing as hypothesized. To test this concept, we examined APP processing in LRPdeficient mouse fibroblasts (LRP-/-) with and without FE65 transfection (Fig. 4a). In LRP-expressing cells, FE65 overexpression resulted in an approximate twofold to threefold increase in APPs secretion versus mock transfected cells (Fig. $4 b$ ), consistent with studies in madin-Darby canine kidney cells (Sabo et al., 1999). As reported previously, basal APPs secretion is increased in LRP-deficient cells (Ulery et al., 2000; Pietrzik et al., 2002). 
However, overexpression of FE65 in LRPdeficient cells did not further augment the level of APPs secretion compared with untransfected control cells (Fig. 4b). One explanation for this latter finding is that the FE65 effect on APP processing is lost in the absence of LRP. Alternatively, it is possible that APPs secretion has already reached a plateau in LRP $-/$ - cells and thus cannot be further augmented by FE65. To exclude this possibility, LRP $-/-$ cells expressing FE65 were treated with PMA to stimulate APPs secretion. As seen, PMA treatment resulted in an additional threefold increase in APPs secretion in LRP $-/-$ cells expressing FE65, arguing against the possibility that APPs release has already reach the maximum level in these cells (Fig. 4c).

The above results were obtained from fibroblasts that were deficient in LRP. To examine the behavior of endogenous levels of LRP, we examined HS683 neuroglioma cells, which revealed a puzzling finding after FE65 expression. Indeed, if FE65 is necessary for APP-LRP complexes to form, one would have predicted that FE65 transfection results in more APP-LRP complexes, in which case APPs secretion would have decreased or remained the same rather than increasing, as detected in this case (Fig. $5 a$ ). Thus, the above experiment was repeated with an FE65 construct lacking the second PID domain (FE65 $\Delta$ PID2) that does not interact with APP, and this mutant therefore cannot function as an APP-LRP adaptor molecule. Surprisingly, overexpression of both FE65 and FE65 $\Delta$ PID2 resulted in an approximate twofold increase in APPs secretion compared with mock transfected cells (Fig. 5a). This indicated that overexpression of both FE65 and FE65 PID2 acted in a dominantnegative manner, possibly by reducing the amount of LRP complexed to APP. In other words, it appears that overexpression of FE65 has sequestered the available LRP, resulting in a state resembling LRP deficiency, and accordingly a rise in APPs secretion.

To test this hypothesis, coimmunoprecipitations were performed in APP695-transfected HS683 human neuroglioma cells. In these cells, complexes of APP and endogenous LRP can be recovered by immunoprecipitation (Fig. $5 b$ ), consistent with a previous report (Rebeck et al., 2001). Closer inspection showed that the endogenous LRP recovered from APP immune complexes consisted of both full-length ( $\alpha+\beta$ subunits) and light chain ( $\beta$ subunit) species, indicating that the interaction occurred in the early secretory pathway before furin cleavage of LRP. Interestingly, after overexpression of either FE65 or FE65 $\Delta$ PID2, less APP-LRP complexes were recovered, suggesting that both FE65 constructs result in sequestration of LRP. Consequently, an increase in APPs secretion accompanied this reduction in levels of APP-FE65-LRP complexes.

\section{RNA short interference against FE65 mimics an LRP knock-out}

In view of the above results, one prediction is that reducing endogenous FE65 levels should also disrupt the link between LRP and APP and similarly perturb APP processing. We have shown previously that expression of the N-terminal LRP-CT in LRPdeficient cells restored some of the abnormalities in APP processing, such as APPs secretion, to that seen in LRP-expressing con- b)

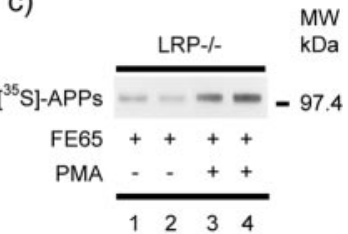
FE65
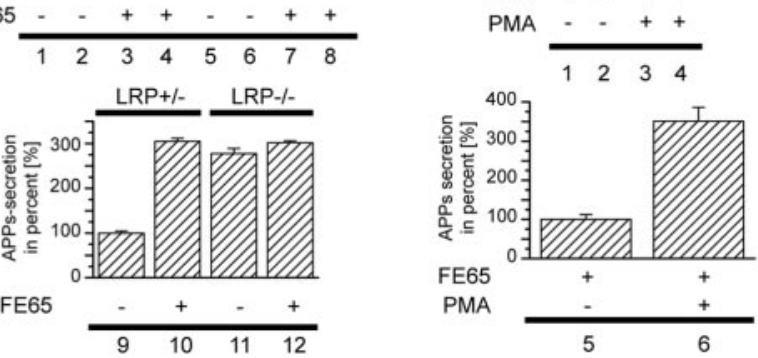

Figure 4. LRP expression mediates FE65-induced APPs secretion. Mouse embryonic fibroblasts lacking the LRP gene secretion after FE65 overexpression (lanes 7,8) compared with untransfected LRP-deficient cells (lanes 5, 6). c, LRP-deficient cells expressing FE65 stimulated with PMA (lanes 3, 4) secrete more APPs than unstimulated cells (lanes 1, 2). MW, Molecular weight.

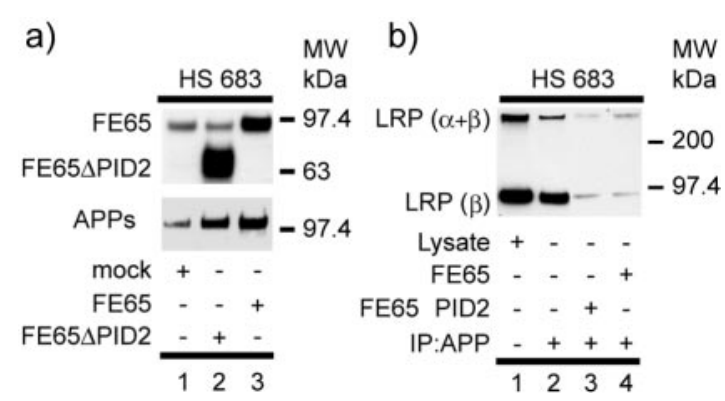

Figure 5. FE65 deletion mutant mediates APP processing. a, HS683 neuroglioma cells stably transfected with APP695 were transiently transfected with FE65 or FE65 $\Delta$ PID2 (top). FE65 levels were immunoblotted with FE65 Ab against the last 15 amino acids of FE65. APPs was immunoprecipitated using the monoclonal antibodies $1 \mathrm{G} 7-5 \mathrm{~A} 3$, and the samples were immunoblotted with an APP pAb (863) as described in Materials and Methods. APPs secretion is significantly increased after transfection with FE65- or FE65 $\triangle$ PID2-transfected cells (lanes 2, 3) compared with control (lane 3). b, APP can be coimmunoprecipitated with LRP from HS683 cells expressing APP695. Using $26 D 6$ Ab, LRP is present in APP immunoprecipitates (lane 2). Corresponding lysate is shown in the left lane (lane 1). Note that both full-length $(\alpha+\beta)$ and furin-cleaved $\beta$-subunit of LRP are detected in APP immunoprecipitates using the anti-LRP Ab 1704 for Western blot analysis. LRP immune complex was significantly decreased after transfection with FE65 or FE65 $\Delta$ PID2 (lanes 3, 4) compared with control (lane 2). MW, Molecular weight; IP, immunoprecipitation.

trol cells. Accordingly, LRP-deficient fibroblasts with or without LRP-CT expression were treated with FE65 RNA short interference (RNAsi) to lower FE65 levels (Fig. 6). RNAsi lowered FE65 by $\sim 80 \%$ (Fig. 6 , bottom panel), and this was accompanied by a twofold increase in APPs secretion in LRP-CT cells (Fig. 6, top panel). However, the reduction in FE65 by RNAsi did not result in altered APPs secretion in the absence of LRP. This result again is consistent with the concept that FE65 is the adaptor molecule that links LRP to APP, and loss of either FE65 or LRP, the latter by genetic deficiency or sequestration by FE65, produces the same abnormal phenotype in APP processing.

\section{Stabilization of APP intracellular domain by FE65 is LRP independent}

As reported by a number of laboratories, FE65 is known to stabilize APP intracellular domain (AICD) and, in doing so, allow the 


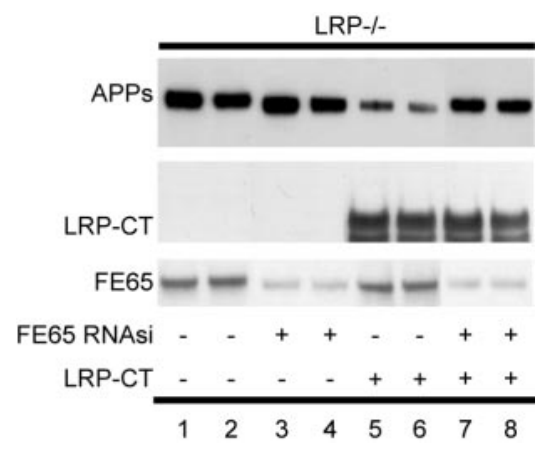

Figure 6. FE65 RNAsi treatment increased APPs secretion. Mouse embryonic fibroblasts lacking the LRP gene (LRP $-/-$ ) and corresponding LRP - / - cells expressing the truncated LRP-CT construct were incubated with FE65 RNAsi constructs for $6 \mathrm{~d}$. Transfected LRP-CT levels were immunoblotted with LRP (-terminal Ab 1704 (middle panel). Endogenous FE65 levels were immunoblotted with FE65 Ab against the last 15 amino acids of FE65 (bottom panel). APPs was immunoprecipitated using the monoclonal antibodies $1 \mathrm{G} 7-5 \mathrm{~A} 3$, and the samples were immunoblotted with an APP pAb (863) as described in Materials and Methods (top panel). APPs secretion is significantly increased after FE65 RNAsi treatment in LRP - / - cells expressing the LRP-CT (lanes 7, 8) compared with controls (lanes 5, 6). APPs secretion remains unaltered in LRP - / - cells after FE65 RNAsi treatment (lanes 3, 4) compared with controls (lanes 1, 2).

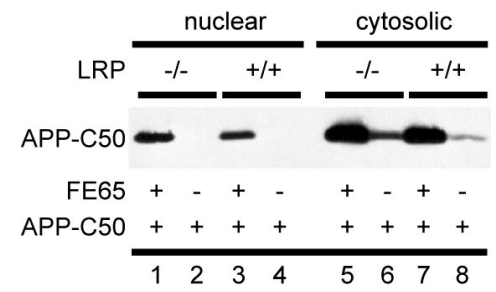

Figure 7. Stabilization of AICD by FE65 is LRP independent. Transfected APP-C50-Myc was immunoprecipitated using an anti-Myc Ab from CHO cell line 13-5-1 deficient in LRP (lanes 1,2, 5, 6) and LRP expressing CH0-K1 control cell line (lanes 3, 4, 7, 8). APP-C50-Myc was detected using the CT15 anti-APP Ab. Note that cells lacking LRP (13-5-1) show no significant difference in APP-C50-Myc expression neither in the cytoplasmic fraction nor in the nuclear fraction compared with LRP-expressing control CHO-K1 cells.

translocation of AICD to the nucleus, the latter presumably to activate downstream nuclear transcription (Kimberly et al., 2001; Ni et al., 2001; Kinoshita et al., 2003). In view of the role of FE65 in linking APP and LRP, we asked whether LRP plays a role in the translocation of AICD to the nucleus. Therefore, an APP construct consisting of the last 50 amino acids (APP-C50) was transfected into LRP-deficient and control cells, and the stability and nuclear localization of AICD was determined with or without FE65. When expressed alone, low amounts of the APP-C50 fragment were present in the NP-40-soluble cytosolic fraction, but it was undetectable in the nuclear SDS-soluble fraction (Fig. 7). As expected, coexpression of FE65 markedly stabilized APP-C50 in both NP-40 and SDS fractions. Interestingly, the same stabilization of APP-C50 after FE65 transfection was observed in LRPdeficient cells in a manner very similar to control LRP-expressing cells. This result therefore provides evidence that LRP does not play a direct role in the translocation of the C-terminal fragment of APP to the nucleus.

\section{Discussion}

LRP is a multifunctional receptor, of which the physiological roles have extended far beyond lipid transport. Recent studies have shown that LRP also plays an important role in mediating APP processing through unclear mechanisms (Ulery et al., 2000; Pietrzik et al., 2002). A number of proteins interact with the cytoplasmic domains of APP and LRP, but the specific functions related to these interactions remain to be elucidated. Fe65 is an excellent candidate to serve as an adaptor or scaffold protein, because it is the only molecule that is known to bind both APP and LRP via distinct domains and colocalize with both APP and LRP (Trommsdorff et al., 1998; Kinoshita et al., 2001). Despite these observations, biochemical evidence supporting an interaction of the three molecules both physically and functionally has been lacking. The current study was designed to test the hypothesis that FE65 does function as an adaptor protein between APP and LRP and that this interaction plays a physiological role in APP processing. Our study provided strong evidence that FE65 does indeed link APP to LRP. Furthermore, our results suggested that the APP-FE65-LRP complex formation regulates APPs secretion.

Through yeast 2-hybrid-defined interactions, it was first hypothesized by Trommsdorff et al. (1998) that FE65 acts as a linker between LRP and APP. FE65 has two colinear PID binding domains that bind LRP and APP, respectively. Thus, the concept that FE65 can act as a scaffold linking APP and LRP is very attractive, although no biochemical data have been provided by these or other investigators. However, LRP has two NPXY motifs that can theoretically interact with FE65. Thus, whether FE65 interacts exclusively with one or the other NPXY motif within the cytoplasmic domain of LRP remains unclear. FE65 is thought to bind to the proximal NPXY motif of LRP, because murine disabled binds to the second NPXY motif of LRP (Trommsdorff et al., 1998). Surprisingly, our studies suggested that FE65 interacts with both NPXY motifs in the cytoplasmic domain of LRP. Specifically, the two LRP-CT constructs deleting one or the other NPXY motif ( $\Delta 1$ and $\Delta 2)$ still associated with FE65. Only by deleting both NPXY motifs (LRP-CT $\Delta 1 \Delta 2$ ) the interaction between FE65 and LRP was lost. Whether these two NPXY sites are functionally equivalent was not addressed in our study. In view of our previous results demonstrating that the distal NPTY motif appeared to be critical for APP processing, it may be that the interaction with second NPTY motif between FE65 and LRP is physiologically more important (Pietrzik et al., 2002).

It was shown previously by confocal microscopy-based FRET techniques that APP695 and LRP are colocalized at the cell surface. Using the same approach, it was subsequently reported that APP and FE65 as well as LRP and FE65 are also in close proximity to each other (Kinoshita et al., 2001). In this study, we provided the biochemical evidence to support the concept that FE65 indeed links APP and LRP in a tripartite protein complex. This was demonstrated by two approaches. First, in pull-down assays, an APP C-terminal fusion protein was able to precipitate cellassociated LRP only when intact FE65 was also expressed but not a FE65 construct lacking the second PID motif. This indicated that the two PID domains, which interact independently with APP and LRP, are required presumably to bridge APP with LRP. Second, LRP C-terminal fusion protein was able to pull down in vitro translated APP695 only when FE65 was also cotranslated but not when FE65 was omitted. Because the latter in vitro experiment was performed with essentially only three components, the results suggested that APP, FE65, and LRP comprise a trimeric complex. Third, APP-LRP complexes can be coimmunoprecipitated in HS683 cells but interestingly, the level of APP-LRP immune complexes was decreased by overexpression of either FE65 or mutant FE65 5 PID2. Together, the findings provided strong evidence that FE65 alone is sufficient to function as a scaffold for APP to complex with LRP.

Since the publication by Trommsdorff and colleagues, it has 
been speculated that the binding of FE65 to APP and LRP somehow mediates APP processing (for review, see De Strooper and Annaert, 2000), but this idea has not been formally tested. Therefore, several experiments that either increased or decreased the levels of FE65 were designed to test the hypothesis that regulation of APP processing by LRP occurs via the cytoplasmic binding of FE65. We first postulated that overexpression of FE65 should influence APP processing by altering the levels of APP-LRP complex. Indeed, FE65 overexpression increased APPs secretion in fibroblasts and neuroglioma cells. Interestingly, when FE65 expression was repeated in cells deficient in LRP, the increase in APPs secretion was lost. This implied that the FE65 effect on APPs secretion was dependent on LRP, or presumably the level of APP-LRP complex. We also speculated that if an increase in APPFE65-LRP complex formation augments APPs secretion, then a disruption of this complex should result in a decrease of APPs secretion. Surprisingly, when a C-terminal-deleted FE65 construct lacking the second PID (APP binding) domain was expressed, increased APPs secretion similar to that seen with overexpression of wild-type FE65 construct was observed. In HS683 cells, this overexpression of FE65 is associated with decreased levels of APP-LRP immune complexes. These results indicated that intact and mutant FE65 both diminish APP-LRP complex formation, resulting in altered APP processing. Recalling that LRP deficiency also increases APPs secretion several-fold (Ulery et al., 2000; Pietrzik et al., 2002), we therefore interpreted the results to indicate that overexpression of either intact or mutant FE65 construct must have sequestered endogenous LRP away from APP, thus producing a phenotype similar to LRP deficiency. Interestingly, this mechanism also provides an explanation for the recent observation that FE65L overexpression reduced the levels of APP C-terminal fragments (Chang et al., 2003). Although the investigators showed quite convincingly that increased $\gamma$-secretase activity is correlated with the reduced APPCTF levels, our results suggest another mechanism whereby FE65L overexpression similarly sequestered LRP away from APP. This reduction in APP-LRP interaction would lead to a decrease in APP-CTFs by increasing turnover, as we showed previously in LRP-deficient cells (Pietrzik et al., 2002).

Similar results consistent with our hypothesis that APP-FE65LRP complex formation is important for APP processing were obtained when FE65 levels were reduced. As mentioned, APPs secretion is elevated in LRP-deficient cells, but this abnormality can be rescued by expressing a truncated LRP construct, LRP-CT. However, the effect of LRP-CT was lost when the cells were treated with RNAsi to lower endogenous FE65 levels. Indeed, the reduction of FE65 by RNAsi treatment resulted in exactly the same phenotype as LRP deficiency. This indicated that lowering FE65 has the same functional effect as LRP deficiency because of the loss of the adaptor molecule. Thus, APP-LRP interaction can be modulated by either increasing or decreasing FE65 levels. Together, these results suggest that in many experimental settings, LRP is the limiting factor for the APP-FE65-LRP complex formation. If true, this would provide a plausible explanation as to why overexpression of FE65 in different cells results in rather disparate outcomes with regard of APP processing (Guenette et al., 1999, 2002; Sabo et al., 1999; Ando et al., 2001; Tanahashi and Tabira, 2002). We propose that differences in LRP levels within different cell types affect the overall level of APP-LRP complexes after FE65 expression: in some cells, the complexes will be reduced as seen in $\mathrm{CHO}$ cells but in others, the complexes will be increased.

Recently, FE65 has been shown to play a crucial role in stabi- lization and translocation of the AICD fragment into the nucleus and subsequent transcriptional activation (Cao and Sudhof, 2001; Baek et al., 2002; Weggen et al., 2003). In view of the preceding results, we asked whether LRP deficiency influences the FE65-mediated nuclear translocation of AICD. Interestingly, we detected no difference between cells expressing LRP or cells lacking LRP expression in their ability of FE65 to stabilize a C-terminal APP construct (APP-C50) as well as its translocation into the nucleus. Whether the lack of LRP alters the transactivation activity of AICD-FE65 complex in the nucleus, as proposed by Kinoshita et al. (2003), remains unanswered. Nonetheless, our results showed that LRP is required predominantly when APP is in its membrane-associated form, either full-length or as C-terminal fragments but not after $\gamma$-secretase cleavage where AICD is released.

In conclusion, the results presented in this study provide additional insights into the regulation of APP processing by LRP. Previous studies have convincingly demonstrated that FE65 lies in close proximity to APP and LRP (Kinoshita et al., 2001). The results reported in the present study support these findings and clearly demonstrate that FE65 acts as a functional linker between APP and LRP. These findings indicate that LRP and FE65 may play a greater and more varied role in Alzheimer's disease pathogenesis than has been suspected.

\section{References}

Ando K, Iijima KI, Elliott JI, Kirino Y, Suzuki T (2001) Phosphorylationdependent regulation of the interaction of amyloid precursor protein with Fe65 affects the production of beta-amyloid. J Biol Chem 276:40353-40361.

Baek SH, Ohgi KA, Rose DW, Koo EH, Glass CK, Rosenfeld MG (2002) Exchange of N-CoR corepressor and Tip60 coactivator complexes links gene expression by NF-kappaB and beta-amyloid precursor protein. Cell 110:55-67.

Cao X, Sudhof TC (2001) A transcriptively active complex of APP with Fe65 and histone acetyltransferase Tip60. Science 293:115-120.

Chang Y, Tesco G, Jeong WJ, Lindsley L, Eckman EA, Eckman CB, Tanzi RE, Guenette SY (2003) Generation of the beta-amyloid peptide and the amyloid precursor protein C-terminal fragment gamma are potentiated by FE65L1. J Biol Chem 278:51100-51107.

De Strooper B, Annaert W (2000) Proteolytic processing and cell biological functions of the amyloid precursor protein. J Cell Sci 113:1857-1870.

Fitzgerald DJ, Fryling CM, Zdanovsky A, Saelinger CB, Kounnas M, Winkles JA, Strickland D, Leppla S (1995) Pseudomonas exotoxin-mediated selection yields cells with altered expression of low-density lipoprotein receptor-related protein. J Cell Biol [Erratum (1995) 130:1015] 129:1533-1541.

Guenette SY, Chen J, Ferland A, Haass C, Capell A, Tanzi RE (1999) hFE65L influences amyloid precursor protein maturation and secretion. J Neurochem 73:985-993.

Guenette SY, Chang Y, Hyman BT, Tanzi RE, Rebeck GW (2002) Lowdensity lipoprotein receptor-related protein levels and endocytic function are reduced by overexpression of the FE65 adaptor protein, FE65L1. J Neurochem 82:755-762.

Herz J, Strickland DK (2001) LRP: a multifunctional scavenger and signaling receptor. J Clin Invest 108:779-784.

Herz J, Kowal RC, Ho YK, Brown MS, Goldstein JL (1990) Low density lipoprotein receptor-related protein mediates endocytosis of monoclonal antibodies in cultured cells and rabbit liver. J Biol Chem 265:21355-21362.

Kang DE, Pietrzik CU, Baum L, Chevallier N, Merriam DE, Kounnas MZ, Wagner SL, Troncoso JC, Kawas CH, Katzman R, Koo EH (2000) Modulation of amyloid beta-protein clearance and Alzheimer's disease susceptibility by the LDL receptor-related protein pathway. J Clin Invest 106:1159-1166.

Kimberly WT, Zheng JB, Guenette SY, Selkoe DJ (2001) The intracellular domain of the beta-amyloid precursor protein is stabilized by Fe65 and translocates to the nucleus in a notch-like manner. J Biol Chem 276:40288-40292. 
Kinoshita A, Shah T, Tangredi MM, Strickland DK, Hyman BT (2003) The intracellular domain of the low density lipoprotein receptor-related protein modulates transactivation mediated by amyloid precursor protein and Fe65. J Biol Chem 278:41182-41188.

Kinoshita A, Whelan CM, Smith CJ, Mikhailenko I, Rebeck GW, Strickland DK, Hyman BT (2001) Demonstration by fluorescence resonance energy transfer of two sites of interaction between the low-density lipoprotein receptor-related protein and the amyloid precursor protein: role of the intracellular adapter protein Fe65. J Neurosci 21:8354-8361.

Koo EH, Squazzo SL, Selkoe DJ, Koo CH (1996) Trafficking of cell-surface amyloid beta-protein precursor. I. Secretion, endocytosis and recycling as detected by labeled monoclonal antibody. J Cell Sci 109:991-998.

Marquez-Sterling NR, Lo AC, Sisodia SS, Koo EH (1997) Trafficking of cell-surface beta-amyloid precursor protein: evidence that a sorting intermediate participates in synaptic vesicle recycling. J Neurosci 17:140-151.

Ni CY, Murphy MP, Golde TE, Carpenter G (2001) gamma-Secretase cleavage and nuclear localization of ErbB-4 receptor tyrosine kinase. Science 294:2179-2181.

Pietrzik CU, Busse T, Merriam DE, Weggen S, Koo EH (2002) The cytoplasmic domain of the LDL receptor-related protein regulates multiple steps in APP processing. EMBO J 21:5691-5700.

Rebeck GW, Moir RD, Mui S, Strickland DK, Tanzi RE, Hyman BT (2001) Association of membrane-bound amyloid precursor protein APP with the apolipoprotein E receptor LRP. Brain Res Mol Brain Res 87:238-245.

Russo T, Faraonio R, Minopoli G, De Candia P, De Renzis S, Zambrano N (1998) Fe65 and the protein network centered around the cytosolic do- main of the Alzheimer's beta-amyloid precursor protein. FEBS Lett 434:1-7.

Sabo SL, Lanier LM, Ikin AF, Khorkova O, Sahasrabudhe S, Greengard P, Buxbaum JD (1999) Regulation of beta-amyloid secretion by FE65, an amyloid protein precursor-binding protein. J Biol Chem 274:7952-7957.

Sisodia SS, Koo EH, Hoffman PN, Perry G, Price DL (1993) Identification and transport of full-length amyloid precursor proteins in rat peripheral nervous system. J Neurosci 13:3136-3142.

Tanahashi H, Tabira T (2002) Characterization of an amyloid precursor protein-binding protein Fe65L2 and its novel isoforms lacking phosphotyrosine-interaction domains. Biochem J 367:687-695.

Trommsdorff M, Borg JP, Margolis B, Herz J (1998) Interaction of cytosolic adaptor proteins with neuronal apolipoprotein $\mathrm{E}$ receptors and the amyloid precursor protein. J Biol Chem 273:33556-33560.

Ulery PG, Beers J, Mikhailenko I, Tanzi RE, Rebeck GW, Hyman BT, Strickland DK (2000) Modulation of beta-amyloid precursor protein processing by the low density lipoprotein receptor-related protein (LRP). Evidence that LRP contributes to the pathogenesis of Alzheimer's disease. J Biol Chem 275:7410-7415.

Weggen S, Eriksen JL, Sagi SA, Pietrzik CU, Golde TE, Koo EH (2003) Abeta42-lowering nonsteroidal anti-inflammatory drugs preserve intramembrane cleavage of the amyloid precursor protein (APP) and ErbB-4 receptor and signaling through the APP intracellular domain. J Biol Chem 278:30748-30754.

Willnow TE, Moehring JM, Inocencio NM, Moehring TJ, Herz J (1996) The low-density-lipoprotein receptor-related protein (LRP) is processed by furin in vivo and in vitro. Biochem J 313:71-76. 